\title{
Boundary Analysis of Text Recognition
}

\author{
Bhushan Thakare \\ Assistant Professor, \\ Department of Computer \\ Engineering, Sinhgad \\ Academy of Engineering, \\ Pune, India

\section{Vanita Doke} \\ BE Student, Department of Computer Engineering, \\ Sinhgad Academy of Engineering, Pune, India
}

\author{
Rohini Salunke \\ BE Student, Department of \\ Computer Engineering, \\ Sinhgad Academy of \\ Engineering, Pune, India
}

\begin{abstract}
:
In today's world, we see many changes in a technology that reduces the typing time and typing mistake "Autocorrect", which was very helpful for humans, but still we have to type manually for anything that we want to store in writing form. If the document or object is very long or continuously processed, typing it takes large time span, or if we have to type anything similar patterns frequently, automation should bring to reduce time and energy or eg. Number plate detection of vehicle on toll plaza, or big parking areas, on such places number plates of vehicle are manually filled to computers for further processing, this takes time. For Document scanning also manually typing is very time consuming, so document scanning is also done by the help of text recognition using boundary analysis. Our project is based on boundary analysis and its application for text recognition. The first part of the project contains introduction of the image segmentation boundary analysis. In the rest of the part it contains some aspects of optimization of algorithms of the boundary analysis.
\end{abstract}

\section{Keywords:}

Contour analysis; Template generation; Boundary detection; Contour Analysis; Text Recognition

\section{INTRODUCTION}

Contour Analysis allows describing, store, comparing and finding the objects presented in the form of the exterior outlines - contours. It is supposed that the contour contains the necessary information on the object shape. Interior points of the object are not accepted to attention. It restricts area of applicability of algorithms of a CA, but reviewing only contours allows to pass from two-dimensional space of the image - to space of contours and by that to lower computing and algorithmic complexity.CA allows to effectively solve the main problems of a pattern recognition - transposition, turn and a rescaling of the image of object. CA methods are invariant to these transformations. In systems of computer vision, some formats of coding of a boundary are used - the code of Freeman, two-dimensional coding, polygonal coding are most known. But all these formats of coding are not used in a CA.Instead, in a CA the boundary is encoded by the sequence consisting of complex numbers. The CA allows to describe, store, compare and find the objects presented in the form of the exterior outlines - contours. It is supposed that the contour contains the necessary information on the object shape. Interior points of the object are not accepted to attention.

\section{Yogeshwari Raykar \\ BE Student, Department of Computer Engineering, Sinhgad Academy of Engineering, Pune, India}

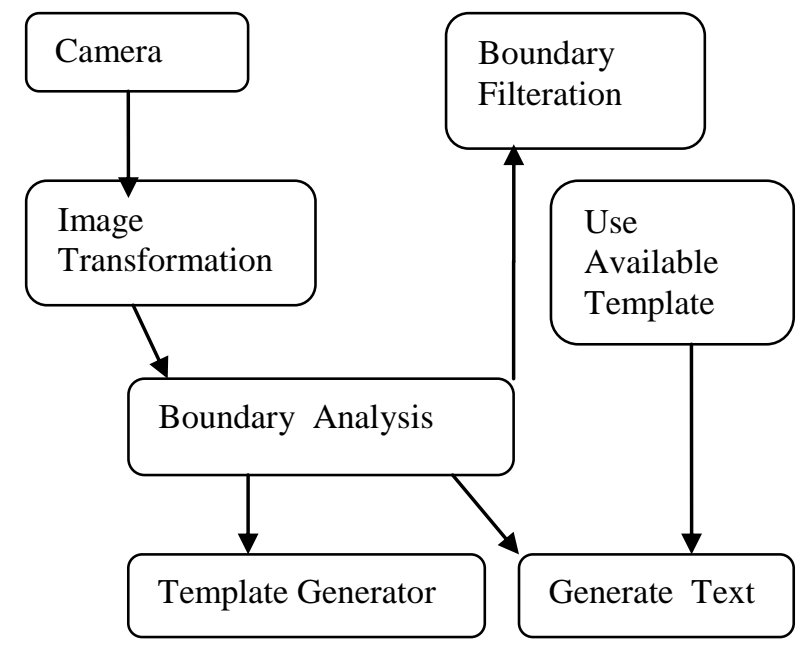

2. LITERATURE SURVEY

In today's world image processing plays an important role, and we can use it for various important aspects, like in our project we are using it for boundary analysis. Current systems which are old and need to upgrade, improve efficiency in the form of time and money. So new technology has to develop to overcome the needs of old technology, to adapt appropriate new technologies from other industries, and to introduce innovations into the image processing and provide people with the new technology which has less consumption of time and energy so as to save their time over manual typing. This paper provides means by which the image processing can develop innovative near-term solutions to meet demands placed on it.

\section{ALGORITHM}

At first, we define an object such as contour. The contour is a boundary of object, a population of points (pixels), and separating object from a background. In systems of computer vision, some formats of coding of a contour are used - the code of Freeman, two-dimensional coding, polygonal coding is most known. But all these formats of coding are not used in a Contour Analysis. Instead, in Contour Analysis the contour is encoded by the sequence consisting of complex numbers. On a contour, the point which is called as starting point is fixed. Then, the contour is scanned (is admissible - clockwise), and each vector of offset is noted by a complex number a+ib. Where $\mathrm{a}$ - point offset on $\mathrm{x}$ axis, and $\mathrm{b}$ - offset on y axis. Offset is noted concerning the previous point.

Owing to the physical nature of three-dimensional objects, their contours are always closed and cannot have selfintersection. It allows to define unambiguously a way of bypass of a contour (to within a direction - on or counter-clockwise). The last vector of a contour always leads to the starting point 
Each vector of a contour we will name elementary vector (EV) and sequence of complex-valued numbers as vector-contour (VC).Contour analysis uses complex-valued coding, because operations over a contour over vector of complex numbers possess remarkable mathematical properties, in comparison with other modes of coding.

Basically, complex coding is close to two-dimensional coding where the contour is defined as a population of the EVs presented in the two-dimensional coordinates. But a difference between operation of scalar product for vectors and for complex numbers - are various.

The Basic Algorithmic Steps are given as follows:

1. Preliminary handling of the image - smoothing, a filtration of noise, a contrast raise

2. Binarization of the image and selection of Boundaries of objects

3. Initial filtration of Boundaries on perimeter, squares, to a crest factor, fractality and so on

4. Coercion of Boundaries to uniform length, smoothing

5. Search of all discovered Boundaries, searching of the template maximum similar to the given Boundary.

We will not consider points 1 and 3, they are specific to application area, and have the small relation to a CA. Further we consider an algorithm body - searching and comparing of boundary with templates. And then - we stop on binarization, coercion to uniform length and smoothing of boundary a little.

\section{EXPERIMENTAL RESULT}

Contour Analysis is done as follows:

1. At first, the image will be transformed to gray-scale.

2. Then it is binarized.

3. Contours are extracted.

4. Contours are filtered on linear parameters (length, square, etc.).

5. Contours are equalized; there is calculation $\mathrm{ACF}$ and ACF descriptors.

6. And then there is maximum suitable template.

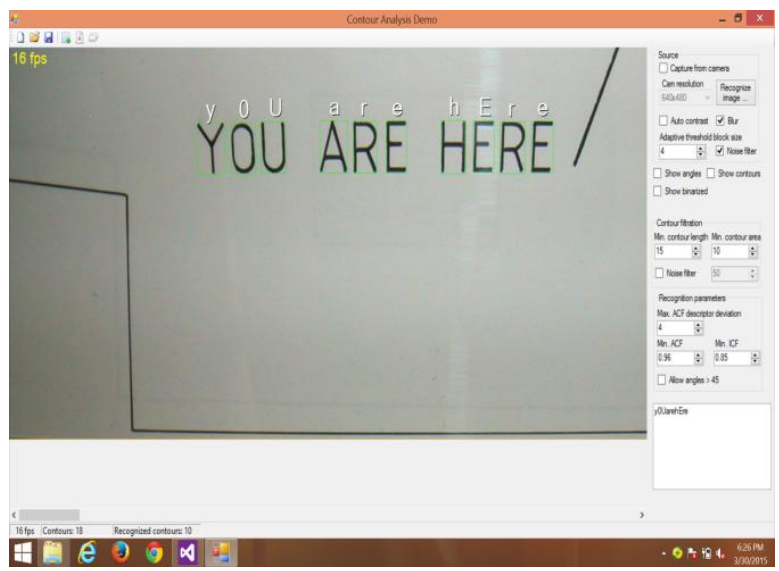

Fig 1. Image capturing
The Image Capturing module is used for capturing image through web cam and display recognized text by considering the properties of images.

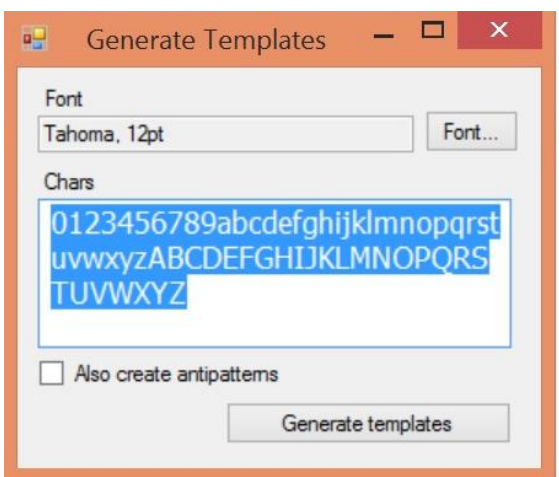

Fig 2. Template Generation

The Template Generator module is used for automatic generation of templates of numerals of a certain font. Except two library projects, there is a demo-example showing operation of library with the webcam. The demo contains tools for creation and editing of templates, recognition tuning, and allows to produce recognition of contours from the webcam, and also allows to create the augmented reality.

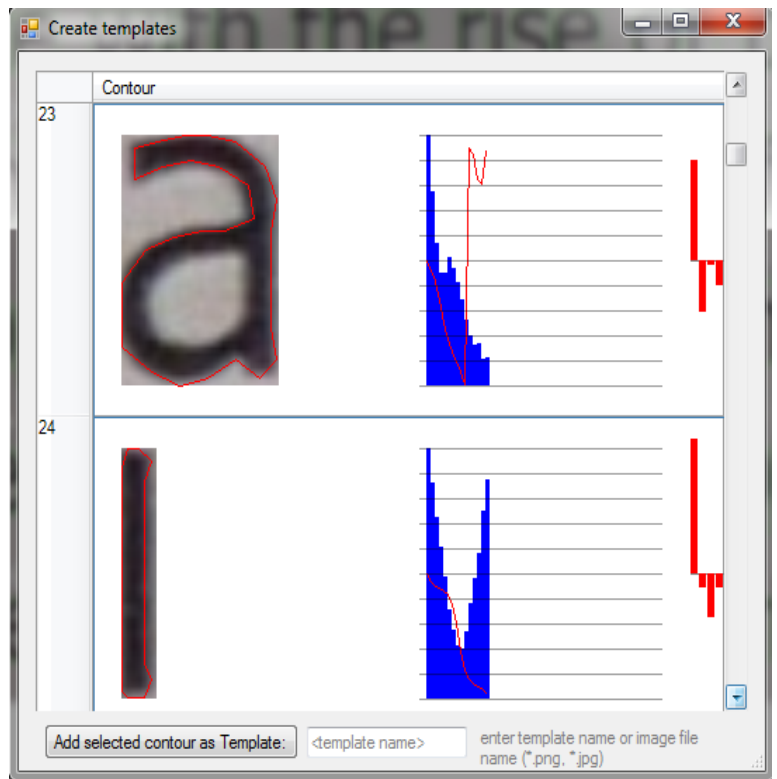

Fig 3. Template Creation

The Template Creation module is used for creating template recognized from image. Since, for another images this created templates are used while matching template.

\section{CONCLUSION}

The main aim of our project was to recognize object by using contour analysis. The increasing number of template results in increase in accuracy. So we can use this contour analysis for many applications like shape recognition, coin recognition, symbol recognition etc. Although contour analysis approach has limitation, our project implemented successfully. Along with updating template, additional feature of finding nearby boundary using contour can be done by using our application. Although our application have some limitations which we can improve by providing noise filtration, blur ratio, contrast etc properties. 


\section{ACKNOWLEDGMENT}

We would like to give our sincere gratitude to our guide $\mathrm{Mr}$. Bhushan Thakare who guided us throughout, to complete this paper.

\section{REFERENCES}

[1] A Contour Analysis Based Technique to Extract Objects using ACF \& ICF.

[2] "A Contour Analysis Based Technique to Extract Objects for MPEG-4.
[3] "The State of the Art in Image Compression Techniques" IJARCCE Journal 2014.

[4] "State of art in Text Recognition Techniques" IJARCCE Journal 2014.

[5] "Boundary and texture Analysis for Image Segmentation" Jitendra Malik, Serge Belongie,Thomas Leung, :Computer science division, University of California at Berkeley, CA 94720-1776, USA. 